\title{
Electron Transport and Ion Acceleration in a Low-Power Cylindrical Hall Thruster
}

\author{
A. Smirnov, Y. Raitses, and N.J. Fisch. \\ Princeton University Plasma Physics Laboratory, P.O. Box 451, Princeton, New Jersey 08543
}

\begin{abstract}
Conventional annular Hall thrusters become inefficient when scaled to low power. Cylindrical Hall thrusters, which have lower surface-to-volume ratio, are therefore more promising for scaling down. They presently exhibit performance comparable with conventional annular Hall thrusters. Electron cross-field transport and ion acceleration in a $2.6 \mathrm{~cm}$ miniaturized cylindrical Hall thruster (100 W power level) are studied through the analysis of experimental data and Monte Carlo simulations of electron and ion dynamics in the thruster channel. The numerical model takes into account elastic and inelastic electron collisions with atoms, electron-wall collisions, including secondary electron emission, and Bohm diffusion. We show that in order to explain the observed discharge current, the electron anomalous collision frequency $v_{B}$ has to be on the order of the Bohm value, $\nu_{\mathrm{B}} \approx \omega_{\mathrm{C}} / 16$. The contribution of electron-wall collisions to cross-field transport is found to be insignificant. The plasma density peak observed at the axis of the $2.6 \mathrm{~cm}$ cylindrical Hall thruster is likely to be due to the convergent flux of ions, which are born in the annular part of the channel and accelerated towards the thruster axis.
\end{abstract}

\section{INTRODUCTION}

The Hall thruster ${ }^{1}$ is a well-studied electric propulsion device at intermediate to high power, but it appears to be promising also for relatively low power primary propulsion on near-Earth missions, ${ }^{2}$ such as orbit transfer and repositioning. The thruster efficiency is defined as $\eta=T^{2} / 2 \mu \mathrm{P}$, where $T$ is the generated thrust, $\mu$ is the supplied propellant flow rate, and $\mathrm{P}$ is the applied electric power. The efficiency of the state-of-the-art kilowatt and subkilowatt conventional Hall thrusters is about $50-60 \%$. The efficiency can be conveniently factorized as: ${ }^{1}$

$$
\eta \approx \frac{I_{i} M}{e \mu} \times \frac{I_{i}}{I_{i}+I_{e}} \times \alpha
$$

where $M$ is a mass of a propellant gas atom, $e$ is the electron charge, $I_{\mathrm{i}}$ and $I_{\mathrm{e}}$ are the electron and ion currents, respectively, and $\alpha$ is the efficiency of ion acceleration. The first fraction in the right hand side of Eq. (1), the propellant utilization, is a measure of how effectively the supplied propellant gas is ionized in the discharge, whereas the second fraction, the current utilization, determines how effectively the electron transport to the anode is suppressed by the applied magnetic field. With all other parameters held constant, the thruster efficiency decreases with increasing electron current. Understanding of the mechanisms of electron transport in the discharge is, therefore, essential for the development of higher efficiency thrusters.

The electrons in Hall thrusters exhibit anomalous cross-field transport: The electron conductivity across the magnetic field is larger than that predicted by the classical electron-atom collision rate. ${ }^{1,3}$ It is believed that two collisional processes contribute to the conductivity enhancement in Hall thrusters: i) electron scattering in electric field fluctuations (anomalous or 'Bohm' diffusion ${ }^{3}$ ), and ii) the electron-wall collisions (the near-wall conductivity ${ }^{4,5}$ ). The electron-wall interaction plays also a very important role by shaping the electron distribution function (EDF) in the thruster channel. In Hall discharge simulations, in order to account for an enhanced electron cross-field transport, the two non-classical conductivity mechanisms are usually incorporated in models in one or another parametric way. In fluid and hybrid fluid-particle models, some investigators impose the anomalous Bohm conductivity inside the channel, ${ }^{6}$ while others use only the near-wall conductivity ${ }^{7}$ or a combination of both Bohm transport and wall collisions. ${ }^{8-12}$ Full particle-in-cell (PIC) simulations ${ }^{13,14}$ reveal turbulence increasing the crossfield transport. Some theoretical studies ${ }^{15,16}$ suggest that due to the non-Maxwellian shape of the EDF in a Hall thruster, electron-wall collisions do not make a significant contribution to cross-field transport. Recently, in a 2-kW Hall thruster operated at low discharge voltage, ${ }^{17}$ in the channel region where the magnetic field was the strongest, 
anomalous fluctuation-enhanced diffusion was identified as the main mechanism of electron cross-field transport. It is important to emphasize here that most of investigations, which addressed the question of the electron conductivity, have been performed for kilowatt and sub-kilowatt thrusters, where the maximal magnetic field strength in the channel is about 100-200 G.

Scaling to low power Hall thrusters requires a thruster channel size to be decreased while the magnetic field must be increased inversely to the scaling factor. ${ }^{1}$ Thus, in general, the rate of electron cross-field transport required to sustain the discharge in a low-power thruster may be different from that in kilowatt thrusters. In other types of low-temperature magnetized laboratory plasmas, variation of the electron cross-field diffusion rate with applied magnetic field $B$ occurs indeed: For example, in Ref. 18, cross-field diffusion coefficient $D_{\perp}$ was observed to approach the Bohm value when $B$ was greater than 2-3 kG, while in $B<1 \mathrm{kG}$ case $D_{\perp}$ was much smaller than the Bohm value.
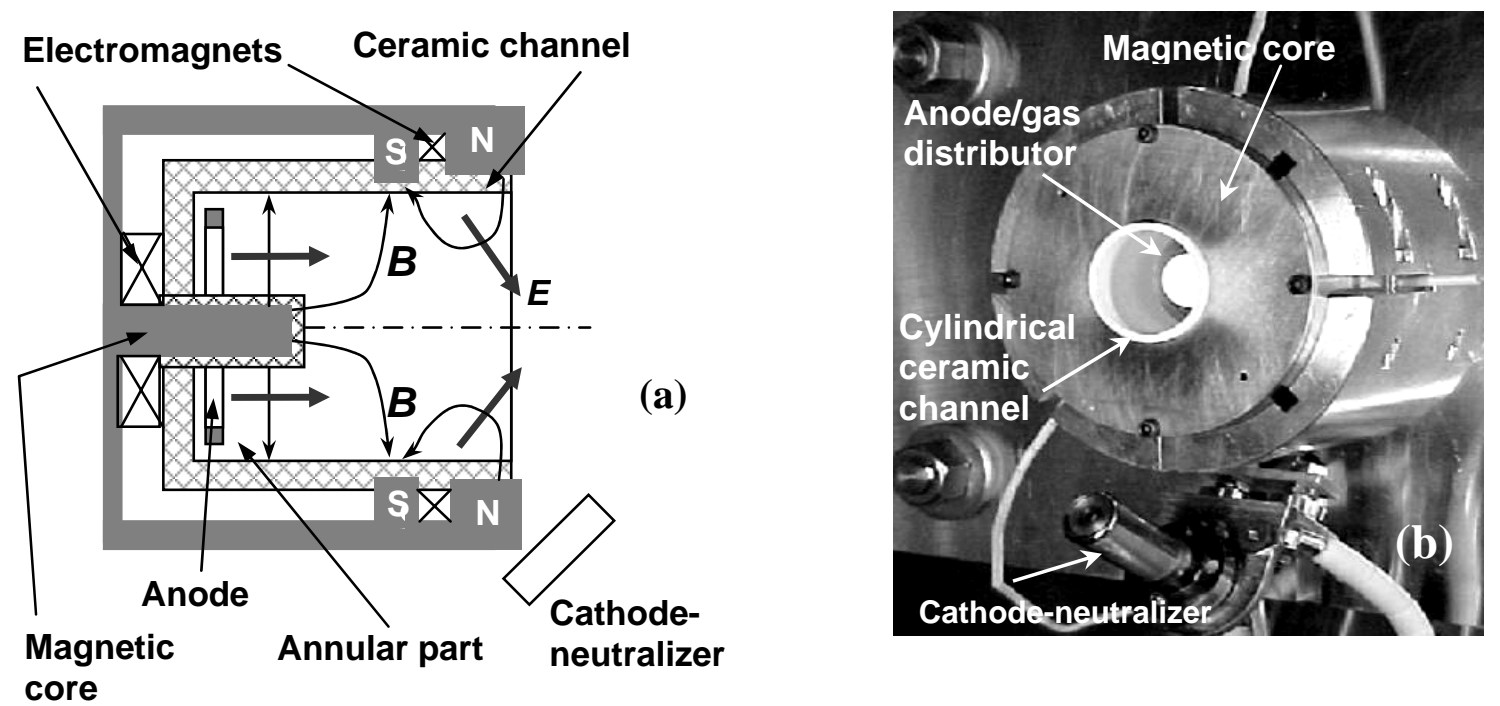

Figure 1. (a) Schematic of a cylindrical Hall thruster. (b) The $2.6 \mathrm{~cm}$ cylindrical Hall thruster.

Increasing the magnetic field while the thruster channel sizes are being reduced is technically challenging because of magnetic saturation in the miniaturized inner parts of the magnetic core. A linear scaling down of the magnetic circuit leaves almost no room for magnetic poles or for heat shields, making difficult the achievement of the optimal magnetic fields. Non-optimal magnetic fields result in enhanced electron transport, power and ion losses, heating and erosion of the thruster parts, particularly the critical inner parts of the coaxial channel and magnetic circuit.

Currently existing low-power Hall thruster laboratory prototypes with channel diameters $2-4 \mathrm{~cm}$ operate at $100-300 \mathrm{~W}$ power levels with efficiencies in the range of $10 \%-40 \% .{ }^{2}$ However, further scaling of the conventional geometry Hall thruster down to sub-centimeter size results in even lower efficiencies, $6 \%$ at power level of about $100 \mathrm{~W} \cdot{ }^{19}$ The low efficiency might arise from a large axial electron current, enhanced by magnetic field degradation due to excessive heating of the thruster magnets, or from a low degree of propellant ionization. Thus, miniaturizing the conventional annular Hall thruster does not appear to be straightforward.

A cylindrical Hall thruster (CHT), illustrated in Fig. 1(a), overcomes these miniaturization problems. ${ }^{20}$ It has been studied, both experimentally and theoretically. ${ }^{21-23}$ The thruster consists of a boron-nitride ceramic channel, an annular anode, which serves also as a gas distributor, two electromagnetic coils, and a magnetic core. What distinguishes this thruster from conventional annular and end-Hall thrusters ${ }^{24}$ is the cylindrical configuration with an enhanced radial component of the cusp-type magnetic field. The cylindrical channel features a short annular region and a longer cylindrical region. The length of the annular region is selected to be approximately equal to an ionization mean free path of a neutral atom. Compared to a conventional geometry (annular) Hall thruster, the CHT has lower surface-to-volume ratio and, therefore, potentially smaller wall losses in the channel. Having potentially smaller wall losses in the channel, a CHT should suffer lower erosion and heating of the thruster parts, particularly the critical inner parts of the channel and magnetic circuit. This makes the concept of a CHT very promising for low-power applications. 
A relatively large $9 \mathrm{~cm}$ diameter version of the cylindrical thruster exhibited performance comparable with conventional annular Hall thrusters in the subkilowatt power range. ${ }^{20}$ It was shown that ion acceleration in the $9 \mathrm{~cm}$ CHT occurs in the cylindrical part of the channel. A miniature $2.6 \mathrm{~cm}$ diameter CHT, in the power range 50-300 W, was shown to have efficiency (15-32\%) and thrust (2.5-12 mN) similar to those of the annular thruster of the same size. ${ }^{21}$ It was found that both the $9 \mathrm{~cm}$ and $2.6 \mathrm{~cm}$ CHTs have unusually high propellant ionization efficiency, compared to conventional Hall thrusters. The propellant utilization, in the case of the $2.6 \mathrm{~cm}$ CHT, could exceed unity, which clearly indicates the presence of multi-charged Xe ions in the ion flux generated by the thruster. In recent work 23, the plasma potential, electron temperature, and plasma density distributions were measured inside the $2.6 \mathrm{~cm}$ CHT. It was found that even though the radial component of the magnetic field has a maximum inside the annular part of the CHT, the larger fraction of the applied voltage, as in the $9 \mathrm{~cm}$ CHT, is localized in the cylindrical region. A significant potential drop was observed also in the plume, where the magnetic field is much weaker than in conventional Hall thrusters.

Ion acceleration in the $2.6 \mathrm{~cm}$ CHT is expected to occur predominantly in the longitudinal direction and towards the thruster axis. Therefore, the CHT, having lower surface-to-volume ratio as compared with conventional Hall thrusters, should suffer lower erosion of the channel walls due to fast ion bombardment. The distribution of plasma density in the cylindrical part of the $2.6 \mathrm{~cm}$ CHT appears to be very non-uniform in the radial direction, with plasma density at the thruster axis is about 4-8 times larger than near the outer channel wall. One possible explanation is that the plasma density spike at the thruster axis might be a manifestation of the convergent ion flux [23].

What the present study offers is a means of understanding the phenomena observed in the cylindrical hall thruster. To study electron transport in the channel region of the $2.6 \mathrm{~cm}$ CHT, a Monte Carlo (MC) code was developed. ${ }^{25}$ The numerical model takes into account elastic and inelastic electron collisions with atoms, electronwall collisions (backscattering, attachment, and secondary electron emission), and Bohm diffusion. The particle tracing part of the code can be used also to simulate ion acceleration in the thruster. Numerical simulations of the plasma discharge in the $2.6 \mathrm{~cm}$ CHT were carried out using the developed MC code. Specifically, the performed simulations addressed two physical questions: i) What rate of electron cross-field diffusion could possibly explain the observed discharge current, and ii) What physical mechanisms bring about the sharp plasma density peak at the thruster axis.

\section{EXPERIMENTS}

The results of comprehensive experimental investigations of the $2.6 \mathrm{~cm} \mathrm{CHT}$ are given in Refs. 21-23. Here, we describe briefly the thruster magnetic field and summarize the experimental results relevant to the purposes of the present study only.

The $2.6 \mathrm{~cm}$ CHT is shown in Fig. 1(b). The total length of the channel is $2.2 \mathrm{~cm}$, the annular region is approximately $0.6 \mathrm{~cm}$ long. The outer and the inner diameters of the channel are $2.6 \mathrm{~cm}$ and $1.4 \mathrm{~cm}$, respectively. The magnetic circuit consists of two coils connected to separate power supplies. The currents in the coils are counterdirected to produce a cusp magnetic field with a strong radial component in the channel.
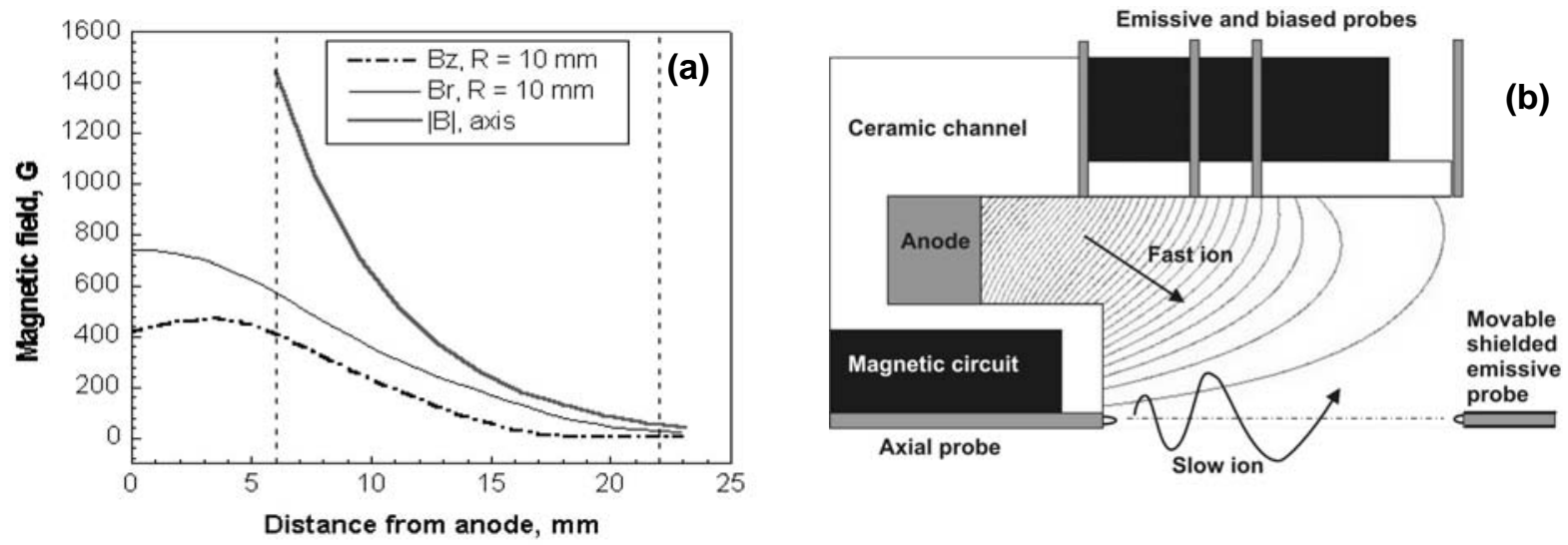

Figure 2. (a) Magnetic field profiles in the $2.6 \mathrm{~cm}$ CHT. $I_{\text {back }}=2.5 \mathrm{~A}, I_{\text {front }}=-1 \mathrm{~A}$. Dashed lines at $\mathrm{z}=6 \mathrm{~mm}$ and $\mathrm{z}=22 \mathrm{~mm}$ show the edge of the annular channel part and the thruster exit, respectively. (b) Probe setup used in the experiments. Magnetic field distribution is given for the same coil currents as in Fig 2(a). Fast and slow ion trajectories are also indicated. 
The magnetic field profiles in the $2.6 \mathrm{~cm}$ CHT are shown in Fig. 2(a). The radial component $B_{r}$ of the magnetic field reaches its maximum near the anode and then reduces towards the channel exit. Although the axial component $B_{z}$ is also strong, the magnetic field in the annular part of the channel is predominantly radial, the average angle between the field line and the normal to the walls is about $30^{\circ}$ [see Fig. 2(b)]. Magnetic field has a mirror-type structure near the thruster axis, with the maximum B 1400 G at the central ceramic piece wall.

The analysis presented in this article was performed for the magnetic field distribution shown in Fig.2 and the following discharge conditions: Xe flow rate $\mu=0.4 \mathrm{mg} / \mathrm{s}$, discharge voltage $U_{\mathrm{d}}=250 \mathrm{~V}$, discharge current $I_{\mathrm{d}} \approx 0.6$ A. Under such conditions, the propellant utilization in the $2.6 \mathrm{~cm} \mathrm{CHT}$ is about 1 , and the current utilization is approximately equal to $0.5 .^{21}$
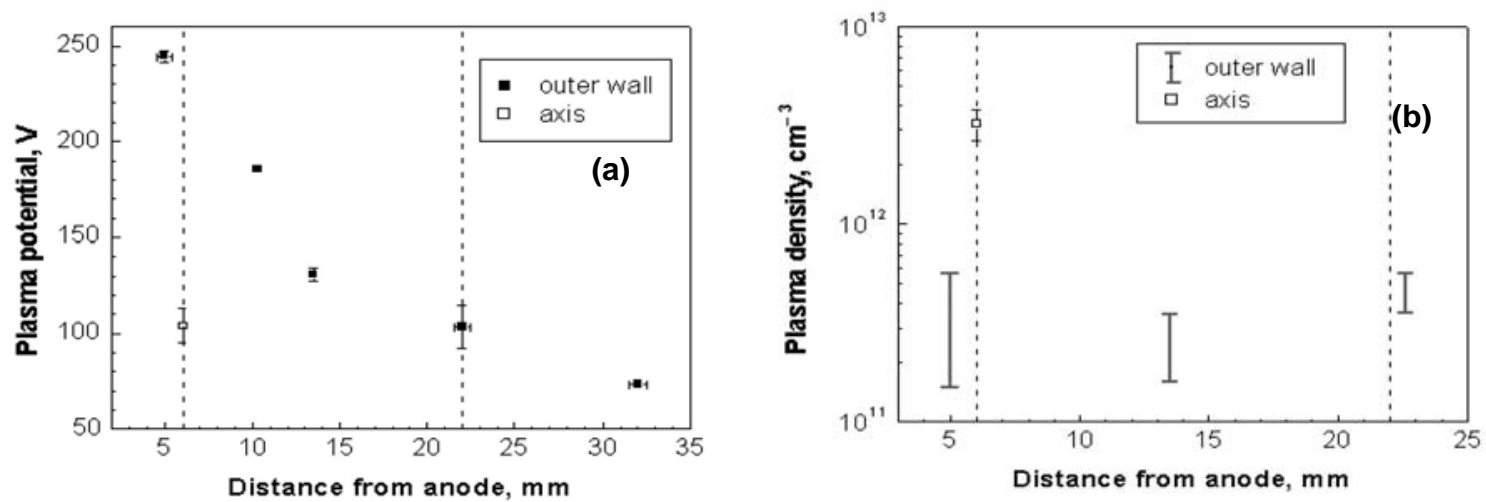

Figure 3. Plasma potential (a) and plasma density (b) profiles in the $2.6 \mathrm{~cm}$ CHT [23]. Dashed lines at $\mathrm{z}=6 \mathrm{~mm}$ and $z=22 \mathrm{~mm}$ show the edge of the annular channel part and the thruster exit, respectively. In (a), Y-axis error bars represent the entire statistical spread of the measured data. For plasma density measurements near the outer channel wall (b), only the intervals, in which the real values of the plasma density are located, can be given.

The distribution of plasma potential $\phi$, electron temperature $T_{\mathrm{e}}$, and plasma density $N_{\mathrm{e}}$ inside the $2.6 \mathrm{~cm}$ CHT was studied by means of stationary and movable floating emissive and biased Langmuir probes. ${ }^{23}$ The probe setup used in the experiments is shown in Fig. 2(b). Measurements were done at the outer channel wall (at four axial locations: $\mathrm{z}=5,10.3,13.5$, and $22 \mathrm{~mm}$ ), as well as at the thruster axis. The results of the probe measurements are shown in Fig. 3. The potential drop in the $2.6 \mathrm{~cm}$ CHT is localized mainly in the cylindrical part of the channel and beyond the thruster exit, in the plume. The potential variation along the thruster axis between the central ceramic piece and the channel exit is insignificant. Its maximum possible value is within the data spread of the measurements, which is about $25 \mathrm{~V}$. Much larger potential drops along the magnetic field lines were observed in the Kaufman ion source [24], which has a mirror-type magnetic field distribution similar to that in the central part of the CHT.

Due to a rather large uncertainty of the plasma density measurements, it was possible to determine only the interval, in which the real value of $N_{\mathrm{e}}$ was located. The variation bars in Fig. 3(b) span between the upper and the lower estimates of $N_{\mathrm{e}}$ obtained in the experiments. ${ }^{23}$ The plasma density in the $2.6 \mathrm{~cm}$ CHT has a prominent peak at the thruster axis: $N_{e}$ at the axis is 4-8 times larger than in the annular part of the channel.

\section{MC CODE DESCRIPTION}

The comprehensive description of the MC code is given elswhere. ${ }^{25}$ In the present paper, we only outline the main code's features and the assumptions we make when performing the numerical simulations.

\section{A. Geometry and fields}

The MC code in the present realization is used to simulate the charged particles dynamics in the channel of the $2.6 \mathrm{~cm}$ CHT. The modeled discharge volume is bounded axially by the anode and the thruster exit plane and radially by the channel walls. The particle trajectories are traced in the given electric and magnetic fields, which are assumed to be azimuthally symmetric. The magnetic field distribution for a given arrangement of the magnetic circuit is simulated using the commercially available Field Precision software. ${ }^{26}$ 
The electric field distribution is obtained from the experiments assuming that the magnetic field surfaces are equipotential. We assign the measured potential values to the magnetic field lines sampled by the corresponding probes [see Fig. 2(b)]. Between the locations of the probes plasma potential $\phi(z, R)$ is assumed to vary linearly with magnetic flux function $\psi(z, R), \phi(z, R) \propto \psi(z, R)$. The anode's surface is equipotential with $\phi=250 \mathrm{~V}$. As suggested by the measurements, the magnetic field line at the thruster axis is assumed to be equipotential as well, and is assigned the potential of $100 \mathrm{~V}$. The sheath potential drop is assumed to be concentrated in the infinitely thin layer near the walls. For the results of measurements shown in Fig. 3, the resultant "tailored" plasma potential profile is plotted in Fig. 4. All numerical simulations were done for this distribution of the plasma potential.

The described "tailoring" procedure for the plasma potential profile does not take into account: i) possible variation of the plasma potential along a magnetic field line, ii) near-wall pre-sheath potential drop, and iii) nearanode sheath potential drop, which value and sign depend on the thruster operating conditions. ${ }^{27}$ The most pronounced deviations of equipotentials from the magnetic field surfaces are expected to occur near the exit plane of the 2.6 CHT, where the electrons are only weakly magnetized, and also in the near-axis region of the cylindrical part of the channel, where the plasma potential might be determined by the convergent ion flux. However, the characteristic magnitude of plasma potential variations that might occur is on the order of $T_{\mathrm{e}}$, which is much smaller than the overall potential drop in the channel. On the other hand, in the present work, we are interested in the gross structure of the plasma discharge only, and the conclusions that we make are quite insensitive to the details of the plasma potential distribution.
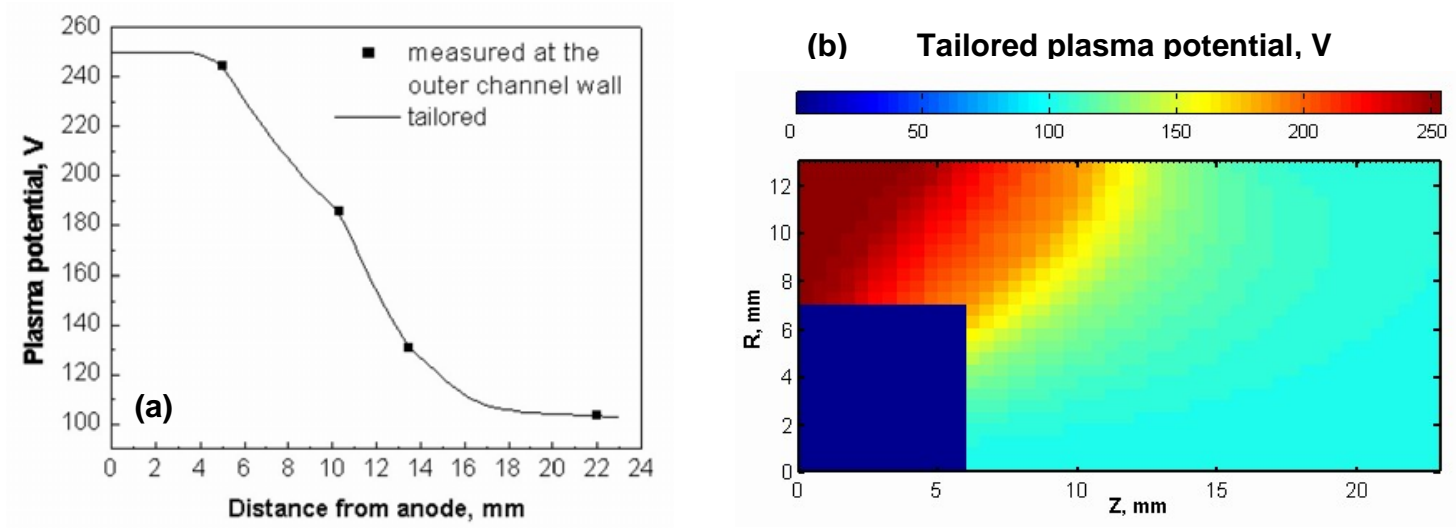

Figure 4. (a) Plasma potential profile along the outer channel wall, measured (symbols) and "tailored" (line). Between the measurement points, plasma potential $\phi(z, R)$ is assumed to be proportional to magnetic flux function $\psi(z, R)$. (b) Distribution of the "tailored" plasma potential in the channel.

\section{B. Particle tracing}

In the MC simulations charged particle trajectories are integrated in 3D-3v (three dimensions in configuration space, three dimensions in velocity space). Newton's equations of motion are resolved using a modification of the explicit leap-frog scheme by Boris. ${ }^{28,29}$ The time step of integration $\Delta t=0.1 / \omega_{\mathrm{c}}$ was used in simulations, where $\omega_{\mathrm{c}}$ is the particle gyrofrequency (for electrons, $\Delta t \sim 3 \times 10^{-12} \mathrm{~s}$ ).

We apply the MC technique ${ }^{30}$ to simulate electron collisions, which include collisions with neutral Xe atoms (elastic scattering, excitation, and single ionization), with channel walls (attachment, backscattering, and secondary electron emission [SEE]), and with electric field fluctuations (anomalous or "Bohm" diffusion). To treat MC collisions, the numerically efficient null-collision method ${ }^{31}$ is implemented in the code. The electron energy and velocity after a collision are determined according to standard scattering relations. ${ }^{32}$

We imposed an anomalous Bohm conductivity inside the channel in order to account for fluctuation-enhanced electron transport. It was assumed that electrons scatter primarily in the azimuthal fluctuations of the electric field. When an electron undergoes a collision with the electric field fluctuation, the perpendicular, with respect to $\mathbf{B}$, electron velocity component is assumed to scatter isotropically. The parallel velocity component does not change. Thus, the guiding center of the electron orbit gets a random shift in the plane perpendicular to $\mathbf{B}$ on the order of the electron gyroradius. The frequency of Bohm diffusion collisions, $v_{\mathrm{B}}=\kappa_{\mathrm{B}} \omega_{\mathrm{C}} / 16$, where $\kappa_{\mathrm{B}}$ is a fitting parameter that does not depend on the electron energy. It is worth mentioning that for kilowatt and subkilowatt Hall thrusters most 
of the models that impose Bohm conductivity in the channel show that the best agreement between the experimental and simulated data is achieved when $\kappa_{B}$ is less than one, on the order of $0.1-0.4^{8,9,11-13}$

In the electron transport simulations, primary electrons injected from the cathode are assumed to have monoenergetic distribution with $\varepsilon=20 \mathrm{eV}$. Similar energy of electrons injected from the cathode was observed in a low-power conventional Hall thruster. ${ }^{33}$ The primary electrons are launched at the thruster exit, with a uniform distribution of the electron flux across the channel cross section. Most of the electrons injected from the cathode to the $2.6 \mathrm{~cm}$ CHT appear to be confined in a hybrid trap, which is formed by the magnetic mirror on the channel side and by the plasma potential drop on the plume side. Diffusion of these electrons across the magnetic field occurs on a time scale much larger than the bounce time in the trap. ${ }^{25}$ In the numerical simulations, the electron trajectories in the plume are not traced. The electrons reflected by the plume potential drop are reinjected into the channel conserving their energy. The injection location is chosen randomly across the thruster exit cross section, and the injection velocity is distributed isotropically.

The electrons are followed successively one after another until both primary electrons and secondary ones (the latter being generated due to ionization and secondary electron emission from the walls), either reach the anode or get attached to the walls. Electron distribution function (EDF) is determined in $z-R-\varepsilon$ phase space using the approach developed by Boeuf and Marode. ${ }^{31}$ Electron density and effective electron temperature are determined as the corresponding moments of the EDF. ${ }^{34}$ Numerical experiments showed that about 3000 electrons have to be traced in order to achieve convergence. The average relative error made in calculation of the energy, which electrons gain while diffusing from the exit plane toward the anode, was found to be less than $3 \%$.

The MC code is used also to simulate ion dynamics in the $2.6 \mathrm{~cm}$ CHT. Since the fields distribution is given, the ion simulations are performed separately from the electron simulations. In the present work we are mainly interested in determining the density profile of fast ions in the cylindrical part of the channel. Due to their large mass, ions in a Hall thruster are not magnetized, $r_{i L}>>L$, where $r_{i L}$ is the ion gyroradius and $L$ is the characteristic channel size. The ions are accelerated by the electric field primarily in the direction perpendicular to the magnetic field surfaces. Since ion mean free path $\lambda_{\mathrm{i}}$ is much larger than the characteristic channel size, ions, to zeroth order in $L / \lambda_{\mathrm{i}}$, can be treated as entirely collisionless.

\section{MODELING APPROACH}

The two specific physical questions addressed in numerical simulations were: i) What rate of electron crossfield diffusion can explain the observed discharge current and ii) What mechanisms bring about the sharp plasma density peak at the thruster axis. As mentioned herein, electron and ion simulations were performed separately from each other.

When simulating the electron dynamics, we have three free parameters, namely, fitting parameter $\kappa_{\mathrm{B}}$, which accounts for Bohm diffusion, neutral gas density $N_{\mathrm{a}}$, and plasma-wall sheath potential drop $\phi_{\mathrm{sh}}$. For simplicity, $N_{\mathrm{a}}$ is assumed to be uniform in the entire channel volume, and $\phi_{\mathrm{sh}}$ is assumed to be constant along all the channel walls. Such approximations seem to be acceptable for the purposes of the present study because the main conclusions that we make appear to be quite insensitive to the uniformity of $N_{\mathrm{a}}$ and $\phi_{\mathrm{sh}}$. It is worth mentioning also that physically reasonable results were obtained under similar assumptions by other authors. ${ }^{16,35}$

To match the numeric results with the measurements, for a given $\kappa_{\mathrm{B}}$, we adjust the values of $N_{\mathrm{a}}$ and $\phi_{\mathrm{sh}}$. The choice of $N_{\mathrm{a}}$ and $\phi_{\mathrm{sh}}$ is determined by two experimental constraints: ${ }^{25}$ (i) The electron current to the anode should be twice as large as the electron current injected from the cathode into the thruster, and (ii) The maximum effective electron temperature $T_{\text {eff }}$ should be approximately equal to the measured value of $18 \mathrm{eV}$. Further details regarding the determination of $N_{\mathrm{a}}$ and $\phi_{\mathrm{sh}}$ are given in Ref. 25.

Ion simulations are performed on the basis of the results of electron simulations. Once an electron simulation is done, we obtain a steady-state distribution of the ionization source in the thruster volume. Loading the ions according to the distribution of the ionization source, and tracing them in the manner described in Sec. III.B., we obtain distributions of the ion density and the mean ion energy in the channel. In the present work we are mainly interested in determining the density profile for ions, which are born in the annular part of the channel and accelerated by the electric field towards the thruster axis. Such ions should form a focused stream in the cylindrical part of the channel, which might explain the plasma density spike observed at the axis.

Note that correct description of the ion wall losses requires the knowledge of plasma potential variation in the pre-sheath. Therefore, a straightforward use of the model potential profile with equipotential magnetic field surfaces (see. Sec. III.A) would produce largely overestimated ion current at the thruster exit. In order to parametrically take into account the ion wall losses, we re-normalize the input ionization source so that the ion current at the thruster 
exit becomes equal to the observed value, $\sim 0.3 \mathrm{~A}$. Such re-normalization effectively reduces the number of ions that leave the channel. Since the wall losses for the fast ions that form the focused stream are suspected to be rather weak, re-normalization of the input ionization source is likely to result in underestimation, rather than overestimation, of the fast ion density in the cylindrical part of the channel.

\section{RESULTS AND DISCUSSION}

\section{A. Electron dynamics}

A parametric study of the dependency of plasma parameters distribution on the electron cross-field conductivity was performed. ${ }^{25}$ Numerical simulations were carried out for different values of $\kappa_{\mathrm{B}}$, with $N_{\mathrm{a}}$ and $\phi_{\mathrm{sh}}$ chosen according to the experimental constraints (see Sec. IV). The main results obtained in electron simulations can be summarized as follows:

(i) The maximum electron density is achieved in the annular part of the channel. Although there is a slight elevation of $N_{\mathrm{e}}$ at the thruster axis, its value, as opposed to the results of the experiments, is lower than the density in the annular part of the channel. When $\kappa_{\mathrm{B}}$ is varied, the distribution of the electron density in the channel does not change qualitatively. The characteristic magnitude of $N_{\mathrm{e}}$ decreases when $\kappa_{\mathrm{B}}$ is increased.

(ii) In order to explain the observed plasma density, the electron anomalous collision frequency $v_{\mathrm{B}}$ should be high, on the order of the Bohm value $\nu_{\mathrm{B}} \approx \omega_{\mathrm{C}} / 16$. Thus, the value of Bohm parameter $\kappa_{\mathrm{B}}$, which, for the low-power $\mathrm{CHT}$, gives the best agreement between the simulations and experiments $\left(\kappa_{\mathrm{B}} \sim 1\right)$, is a few times larger than those obtained typically in the modeling of conventional Hall thrusters $\left(\kappa_{\mathrm{B}} \sim 0.1-0.4\right){ }^{8,9,11-13}$ Therefore, the rate of electron fluctuation-enhanced diffusion, which is required to explain the discharge current observed in the CHT, should be higher than that in conventional Hall thrusters. The anomalous electron transport in the CHT is believed to be induced by high-frequency plasma instabilities. Interestingly, in the frequency range below $\sim 100 \mathrm{kHz}$, the $2.6 \mathrm{~cm}$ CHT operates quieter than the annular Hall thruster of the same size. ${ }^{21}$

(iii) Electron-wall collisions deplete the tail of the EDF. The resultant shape of the EDF appears to be biMaxwellian. As $\kappa_{\mathrm{B}}$ (and, consequently, $v_{\mathrm{B}}$ ) decreases, the tail of the distribution function gradually weakens. The general shape of the EDF obtained in simulations appears to be in a good qualitative agreement with the results of work [16], where the EDF in the Hall thruster channel was determined by solving the electron Boltzman equation.

(iv) The electron-wall collisions make an insignificant contribution to the electron current conduction, as compared with the fluctuation-induced electron scattering. The typical average electron-wall collision frequency, $v_{\mathrm{ew}}$, is on the order of $1 \times 10^{7} \mathrm{~s}^{-1}$, while the anomalous collision frequency $v_{\mathrm{B}}$, averaged along a magnetic field line, is about $7 \times 10^{8} \mathrm{~s}^{-1}$. Inequality $v_{\mathrm{ew}}<<v_{\mathrm{B}}$ is satisfied throughout the thruster channel.

\section{B. Ion dynamics}

Due to rather high electron temperature and density, ionization of neutrals injected from the anode occurs mainly in the annular part of the channel. Distribution of ionization source $S(z, R)$ is computed as $S(z, R)=N_{a} \int_{0}^{\infty} \sigma_{i}(\varepsilon) f(z, R, \varepsilon) \sqrt{2 \varepsilon / m} d \varepsilon$, where $m$ is the electron mass. A typical ionization source distribution in the $2.6 \mathrm{~cm}$ CHT is shown in Fig. 5.

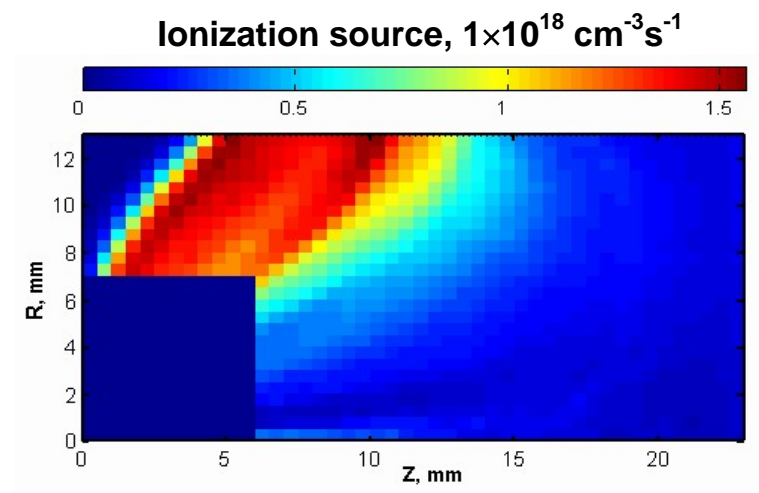

Figure 5. Typical ionization source distribution in the channel of the $2.6 \mathrm{~cm}$ CHT. The solid dark rectangle in the lower left-hand side corner of the picture $(0<z<6,0<R<7)$ represents the cross section of the central ceramic piece. 
Typical computed distributions of the ion density and the mean ion energy in the channel of the $2.6 \mathrm{~cm}$ CHT are shown in Fig. 6. Due to ion acceleration in the direction perpendicular to the magnetic field surfaces, ions generated in the annular part of the channel form a focused flux. This flux, converging at the thruster axis in the cylindrical part of the channel, brings about an ion density spike. As can be seen from Fig. 6(a), the ion density at the thruster axis appears to be more than an order of magnitude higher than near the outer wall.

As follows from calculations of the ion energy distribution function (IDF), the ion density peak at the axis is due mainly to fast ions. In Fig. 7, the IDF at $R=0.25 \mathrm{~mm}$ and $z=15 \mathrm{~mm}$ is shown. The ion population in the near-axis region consists of two ion fractions, namely, slow ions with energy less or equal to about $10 \mathrm{eV}$, and fast ones with broad energy spectrum from $10 \mathrm{eV}$ to about $150 \mathrm{eV}$. Slow ions are those born in the near-axis region. Due to the imposed equipotentiality of the magnetic field surfaces, these ions do not accelerate much. Fast ions are those forming the focused ion flux. As can be shown by integrating the IDF of Fig. 7 in the corresponding limits, the density of the fast ions at the thruster axis is about twice as large as that of the slow ones.

(a)

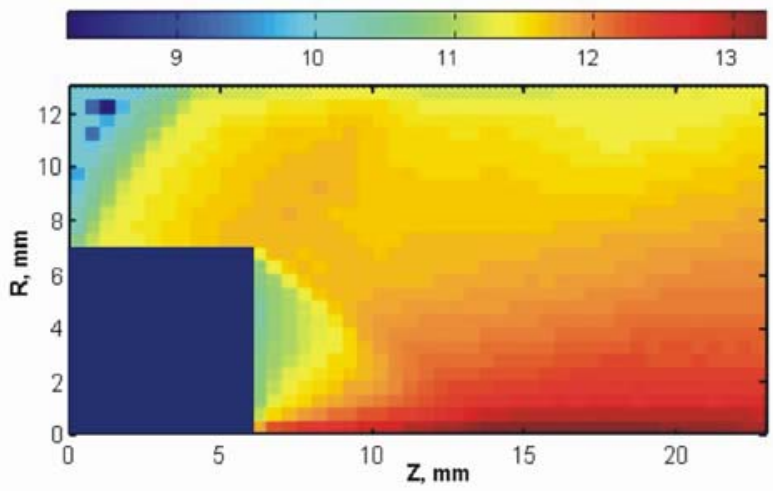

(b)

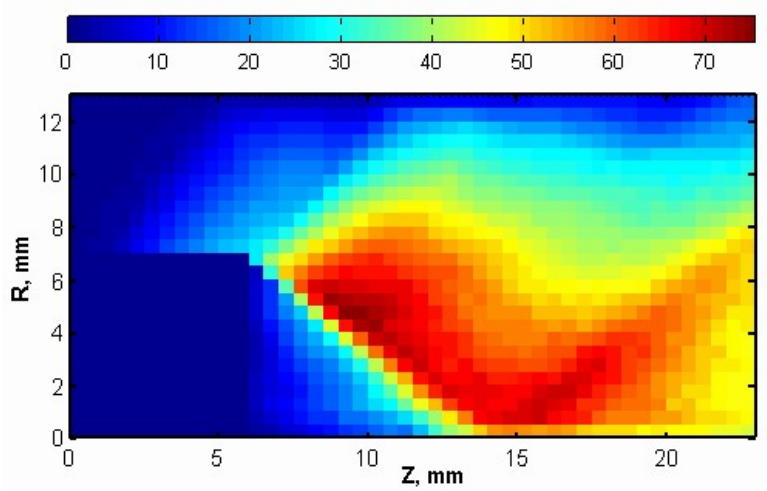

Figure 6. Typical distributions of the ion density (a) and the mean ion energy (b) in the channel of the $2.6 \mathrm{~cm}$ CHT. Ion density is plotted in logarithmic scale. The solid dark rectangle in the lower left-hand side corner of the pictures $(0<z<6,0<R<7)$ represents the cross section of the central ceramic piece.

For the same simulation parameters, the ion density at the thruster axis appears to be more than an order of magnitude higher than the electron density. ${ }^{25}$ In reality, such a severe charge separation in the plasma bulk is, of course, impossible. If the ions tended to outnumber the electrons at the thruster axis, then, in order to preserve plasma quasineutrality, the ambipolar plasma potential would rise, and the electrons would be pulled to the near-axis region to compensate an excessive positive space charge. To attract enough electrons, the magnitude of the potential elevation at the axis should be on the order of the electron temperature. Therefore, the model potential distribution imposed in the present simulations can deviate appreciably from the real one in the cylindrical part of the channel. Implications of this fact are discussed next.

In the vicinity of the geometric 'focal point' of the ion flux $(R=0, \mathrm{z} \approx 15 \mathrm{~mm})$, there should appear a selfconsistent ambipolar potential peak associated with the plasma density elevation. Physically, this situation is similar, for example, to what happens in a gas-dynamic mirror trap (GDMT) with fast ion injection. In GDMTs, fast ion beam injection can cause an increase of the ambipolar potential at the mirror plug, thus improving the trapping of slow (gas-dynamically confined) ions. ${ }^{36}$

A rough estimate of potential peak height $\Delta \phi$ can be obtained by using the Boltzman distribution for the electrons and slow ions in the near-axis region of the CHT. Let's denote the electron density near the central ceramic piece wall as $N_{0}$ and assume that fast ions that form the focused flux have energy much larger than $\Delta \phi$. Then the quasineutrality condition at the 'focal point' reads:

$$
N_{0} \exp \left(\frac{e \Delta \phi}{T_{e}}\right)=N_{0} \exp \left(\frac{-e \Delta \phi}{T_{s}}\right)+N_{f},
$$

where $T_{\mathrm{e}}$ and $T_{\mathrm{s}}$ are the electron and the slow ion temperatures, respectively, and $N_{\mathrm{f}}$ is the fast ion density. Since $T_{\mathrm{s}}<<T_{\mathrm{e}}$, the first term in the right hand side of Eq. (3) can be neglected, and the potential peak height is then 


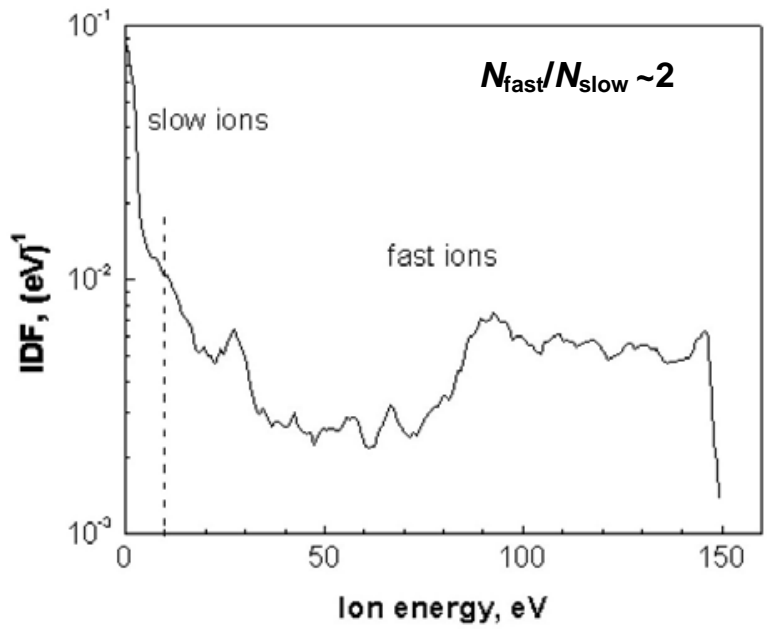

Figure 7. Ion distribution function (IDF) in the near-axis region of the cylindrical part of the channel (at $R=0.25 \mathrm{~mm}$ and $z=15 \mathrm{~mm}$ ). $N_{\text {slow }}$ and $N_{\text {fast }}$ are the densities of slow and fast ions, obtained by integrating the IDF from 0 to $10 \mathrm{eV}$ and from $10 \mathrm{eV}$ to $150 \mathrm{eV}$, respectively.
$\Delta \phi \sim T_{\mathrm{e}} \ln \left(N_{\mathrm{f}} / N_{0}\right)$. (The result of this estimate must not be confused with the simulation results shown in Figs. (6), (7). In our simulations, the near-axis region is equipotential and, therefore, the density of slow ions at the axis (see Fig. 7) is less but comparable with that of fast ions.)

The implications of the potential peak existence at the thruster axis might be very important for the physics of the CHT. The potential peak should reflect slow ions generated near the central ceramic wall back into the thruster. This, on the one hand, might increase the residence time of slow ions in the discharge. A larger ion residence time increases the probability of ionization to $\mathrm{Xe}^{+2}$ charge state and, thus, could help to explain the enhanced propellant utilization observed in the CHTs. ${ }^{22}$ On the other hand, the reflected slow ions impinging the central ceramic piece might cause additional wall erosion. A similar mechanism enhancing the thruster parts erosion was discussed in work, ${ }^{37}$ where the axial increase of the ambipolar potential was observed in the plume of four clustered low-power Hall thrusters.

\section{CONCLUSIONS}

Scaling to low-power Hall thrusters requires the magnetic field to be increased inversely with length, as the thruster channel size is decreased. In a strong magnetic field of a low-power Hall thruster, the rate of electron crossfield diffusion required to sustain the discharge can differ from that in Hall thruster operating in the conventional kilowatt or subkilowatt power range. Thus, understanding of the mechanisms of electron transport is essential for the development of higher efficiency low-power thrusters and for the scaling to small sizes.

The conventional (annular) Hall thrusters become inefficient when scaled to small sizes because of the large surface-to-volume ratio and the difficulty in miniaturizing the magnetic circuit. Also, the erosion of the walls of a small annular channel can severely limit the thruster lifetime. An alternative approach, which may be more suitable for scaling to low power, is a cylindrical Hall thruster (CHT). Both the $9 \mathrm{~cm} \mathrm{CHT}$, operated in the subkilowatt power range, and the miniature $2.6 \mathrm{~cm} \mathrm{CHT}$, operated in the power range 50-300 W, exhibit performance comparable with conventional annular Hall thrusters of the similar size. Ion acceleration in both CHTs occurs mainly in the cylindrical part of the channel and beyond the thruster exit.

To study electron and ion dynamics in the channel region of the $2.6 \mathrm{~cm}$ CHT, a Monte Carlo code was developed. The numerical model takes into account elastic and inelastic electron collisions with atoms, electron-wall collisions (backscattering, attachment, and secondary electron emission), and Bohm diffusion. The numerical simulations of electron cross-field transport in the $2.6 \mathrm{~cm}$ CHT have been performed and their results have been compared with the measurements. In order to explain the discharge current observed in the $2.6 \mathrm{~cm} \mathrm{CHT}$, the electron anomalous collision frequency $v_{\mathrm{B}}$ has to be high. As opposed to most of the conventional Hall thruster models, which predict the ratio $v_{\mathrm{B}} / \omega_{\mathrm{C}}$ to be on the order of $10^{-2}$, we find that in the $2.6 \mathrm{~cm}$ CHT $\nu_{\mathrm{B}}$ has to be on the order of the Bohm value, $\nu_{\mathrm{B}} \sim \omega_{\mathrm{C}} / 16$. The anomalous cross-field electron transport in the CHT is believed to be induced by high-frequency plasma instabilities.

The electron distribution function (EDF) obtained in the simulations is in good qualitative agreement with the results of Ref. 16: The EDF in a Hall thruster is depleted at high energy due to electron loss at the walls, thus indicating that the contribution of secondary electrons to cross-field transport is likely insignificant.

The plasma density peak observed at the axis of the $2.6 \mathrm{~cm}$ CHT appears to be due to the convergent flux of ions, which are born in the annular part of the channel and accelerated towards the thruster axis. It is suggested that a plasma potential peak appears in the cylindrical part of the channel. Ambipolar trapping of slow ions in the near-axis region of the thruster might explain the enhanced propellant utilization observed in the CHTs. 


\section{ACKNOWLEDGMENTS}

The authors are grateful to Prof. Vladimir Semenov for helpful discussions and comments on this article. This work was supported by grants from AFOSR, DARPA, and USDOE Contract AC02-76CH0-3073.

\section{REFERENCES}

1. I. Morozov and V. V. Savelyev, in Review of Plasma Physics, edited by B. B. Kadomtsev and V. D. Shafranov (Consultants Bureau, New York, 2000), Vol. 21, p. 203.

2. J. Mueller, in Micropropulsion for Small Spacecraft, edited by M.M. Micci and A.D. Ketsdever (AIAA Progress in Astronautics and Aeronautics, 2000), Vol. 187, p. 45.

3. G.S. Janes and R.S. Lowder, Phys. Fluids 9, 1115 (1966).

4. A.I. Morozov and A.P. Shubin, Sov. J. Plasm. Phys. 10, 12 (1984).

5. A.I. Bugrova, A.I. Morozov, and V.K. Kharchevnikov, Sov. J. Plasm. Phys. 16, 849 (1990).

6. E. Fernandez and M.A. Cappelli, Bull. Am. Phys. Soc. 45(7), 166 (2000).

7. S. Barral, K. Makowski, Z. Peradzynski, N. Gascon, and M. Dudeck, Phys. Plasmas 10, 4137 (2003).

8. E. Ahedo, J.M. Gallardo, and M. Martinez-Sanchez, Phys. Plasmas 10, 3397 (2003).

9. M. Keidar, I.D. Boyd, and I.I. Beilis, Phys. Plasmas 8, 5315 (2001).

10. J. Bareilles, G.J.M. Hagelaar, L. Garrigues, C. Boniface, J.P. Boeuf, and N. Gascon, Phys. Plasmas 11, 3035 (2004).

11. J.M. Fife, Ph.D. thesis, Massachusetts Institute of Technology, 1998.

12. J.J. Szabo, Ph.D. thesis, Massachusetts Institute of Technology, 2001.

13. O. Batishchev and M. Martinez-Sanchez, in Proceedings of the 28th International Electric Propulsion Conference, Toulouse, France (Electric Rocket Propulsion Society, Cleveland, OH, 2003), IEPC 03-188.

14. J.C Adam, A. Heron, G. Laval, Phys. Plasmas 11, 295 (2004).

15. L. Jolivet and J.F. Roussel, in SP-465: 3rd Spacecraft Propulsion Conference, Cannes, France (European Space Agency, Noordwijk, The Netherlands, 2000), pp. 367-376.

16. N.B. Meezan and M.A. Cappelli, Phys. Rev. E 66, 036401 (2002).

17. N.B. Meezan, W.A. Hargus, and M.A. Cappelli, Phys. Rev. E 63, 026410, (2001).

18. M. Stanojevic, M. Cercek, T. Gyergyek, and N. Jelic, Contrib. Plasma Phys. 34, 607 (1994).

19. V. Khayms and M. Martinez-Sanches, in Micropropulsion for Small Spacecraft, Progress in Astronautics and Aeronautics, edited by M.M. Micci and A.D. Ketsdever (American Institute of Aeronautics and Astronautics, Reston, VA, 2000), Vol. 187, p. 45.

20. Y. Raitses and N.J. Fisch, Phys. Plasmas 8, 2579 (2001).

21. A. Smirnov, Y. Raitses, and N.J. Fisch, J. Appl. Phys. 92, 5673 (2002).

22. A. Smirnov, Y. Raitses, and N.J. Fisch, J. Appl. Phys. 94, 852 (2003).

23. A. Smirnov, Y. Raitses, and N.J. Fisch, J. Appl. Phys. 95, 2283 (2004).

24. H.R. Kaufman, R.S. Robinson, and R.I. Seddon, J. Vac. Sci. Technol. A 5, 2081 (1987).

25. A. Smirnov, Y. Raitses, and N.J. Fisch, submitted to Phys. Plasmas in May, 2004.

26. http://www.fieldprecision.com

27. L. Dorf, Y. Raitses, N.J. Fisch, and V. Semenov, Appl. Phys. Lett. 84, 1070 (2004).

28. J.P. Boris, in the Proc. 4th Conf. Num. Sim. Plasmas, Naval Res. Lab, Washington, DC, pp. 3-67, Nov.1970.

29. C.K. Birdsall and A.B. Langdon, Plasma Physics Via Computer Simulation (New York, McGraw-Hill, 1985), pp. 120-121.

30. C.K. Birdsall, IEEE Trans. Plasm. Sci. 19, 65 (1991).

31. J.P. Boeuf, E. Marode, J. Phys.D 15, 2169 (1982).

32. L.D. Landau and E.M. Lifshitz, Mechanics (New York, Pergamon Press, 1976), pp.44-47.

33. V. Yu. Fedotov, A.A. Ivanov, G. Guerrini, A.N. Vesselovzorov, and M. Bacal, Phys. Plasmas 6, 4360 (1999).

34. V.E. Golant, A.P. Zhilinsky, I.E. Sakharov, and S.C. Brown, Fondamentals of Plasma Physics (John Wiley \& Sons, New York, 1977), p.155.

35. V. Latocha, L. Garrigues, P. Degond, and J.P. Boeuf, Plasma Sources Sci. Technol. 11, 104 (2002).

36. I.A. Kotel'nikov and D.D. Ryutov, Sov. J. Plasm. Phys. 11, 655 (1985).

37. B.E. Beal, A.D. Gallimore, and W.A. Hargus, Jr., in Proceedings of the 39th Joint Propulsion Conference, Huntsville, $A L$ (American Institute of Aeronautics and Astronautics, Reston, VA, 2003), AIAA 03-5155. 